\title{
Energy dependence of the electroproduction of low mass vector mesons
}

\author{
Khalid M. A. Hamad ${ }^{*}$, Afraa Ammash Kanaan, Mysoon Kamil Bnaya \\ Department of Physics, College of Science, Al-Anbar University, Ramadi, Iraq \\ *E-mail address: khalid_hamad2oo2@yahoo.com
}

\begin{abstract}
The energy dependence of the electroproduction of low mass vector mesons has been analyzed. Two pomeron model is used assuming that the pomeron is a Regge pole. Regge amplitude for the photoproduction processes is extended to include the electroproduction. In this case the residues of the two poles are functions of the virtuality of the photon. These functions are extracted from the experimental data using the chi- square method. A reasonable fit to the data is obtained including these functions in the two pomeron model.
\end{abstract}

Keywords: low mass vector mesons; pomeron model; Regge pole; photon

\section{INTRODUCTION}

Diffractive electroproduction of vector mesons can be represented by the reaction $\gamma^{*} p \rightarrow v p$, where $v=\rho, w, \varphi, J / \psi, \ldots \ldots$ The hard scattering can be reached if one of the variables $Q^{2}$, t and $m_{v}$ is high, with $Q^{2}$ is the mass of the virtual photon, $\mathrm{t}$ is the transferred momentum and $m_{v}$ is the mass of the vector meson. At low $Q^{2}, t$ and $m_{v}$ the process is soft and can be described by a soft pomeron exchanged [1]. The hard process is achieved when one or more of these variables are high, then the QCD calculation can be applied [2-5]. The total cross section can be factorized into $Q^{2}$ and $w$ dependence forms

$$
\sigma_{T}=\sigma_{\gamma^{*} p \rightarrow v p}\left(Q^{2}\right) \sigma_{\gamma^{*} p \rightarrow v p}(w)
$$

where $w$ is the energy of the interaction. Experimentally, the $Q^{2}$ dependence is giving by the form $[6,7]$

$$
\frac{1}{\left(Q^{2}+m_{v}^{2}\right)^{n}}
$$

with $n \sim 2.3$. Most of the properties of the electroproduction of the vector mesons can be reproduced by the QCD calculations in spite of an uncertainty in the overall normalization. Different models have discussed the $Q^{2}$ dependence using different approaches. Some of these models assume that the coupling of the photon-vector meson vertex to the exchanged pomeron is responsible for this dependence [8]. 
Some other models used the $Q^{2}$ in the parameters of pomeron trajectory $[9,10]$. However, the variation of the cross section with $w$ for different values of $Q^{2}$ is not discussed in such types of models. In this paper, a two pomeron model is used to analyze this variation for light vector mesons. The generalization for heavy vector mesons is straightforward. Experimentally, this variation has been parameterized as $w^{\delta}$. For photoproduction [11-19], there is a pattern of variation of the exponent $\delta$ from light to heavy vector mesons. For $\rho$ and $\varphi \quad \delta \cong 0.22$ while the measurement of the $J / \psi$ photoproduction showed a much steeper rise with energy than the light vector mesons with $\delta$ was found to be $0.7-0.8$. It is also found that $\delta$ for $\Upsilon$ is about 1.6. The cross section has a smooth dependence on $w$ for small $\delta$ while it has steep dependence for higher values of $\delta$. This phenomenon has been explained as presence of two pomerons, the soft and the hard one. In the photoproduction the transition from the soft region to the hard region is due to the mass scale. The transition can also be obtained by varying the $Q^{2}$ for electroproduction of $\rho$ and $\varphi$ mesons [20,21]. The $w^{\delta}$ parameterization for $\sigma_{\gamma^{*} p \rightarrow \rho p}(w)$ and $\sigma_{\gamma^{*} p \rightarrow \varphi p}(w)$ for different values of $Q^{2}$ indicates that $\delta$ is changing from 0.22 at small $Q^{2}$ as in the photoproduction and increasing with increasing $Q^{2}$ up to 0.8-1.1 for highest values of $Q^{2}$. For $J / \psi$ the variation of $\delta$ with $Q^{2}$ is small with an average value around 0.8. in the present analysis the pomeron is regarded as a Regge pole so $\delta$ is independent of $Q^{2}$.

\section{THE MODEL}

The calculation of the vertex of coupling of the pomeron to the virtual photon-vector meson vertex is given by [22]

$$
f\left(Q^{2}, 0, m_{v}^{2}\right)=\frac{m_{v}^{2}}{Q^{2}+m_{v}^{2}}\left(1+\frac{1}{\pi^{2}-\ln ^{2}\left(\frac{1+\beta_{2}}{1-\beta_{2}}\right)} \ln ^{2}\left(\frac{\beta_{1}+1}{\beta_{1}-1}\right)\right)
$$

all the parameters are defined in the reference. The cross section is given as

$$
\sigma_{\gamma^{*} p \rightarrow v p}\left(Q^{2}\right)=\left[\frac{\mathrm{m}_{v}^{2}}{\mathrm{Q}^{2}+\mathrm{m}_{v}^{2}} f\left(Q^{2}, 0, m_{\rho}^{2}\right)\right]^{2}
$$

The contribution from the longitudinal photon is given by an equation similar to that in (4) multiplied by $\epsilon \frac{Q^{2}}{m_{v}^{2}}$ with $\epsilon \approx 1$.

Different models indicate that a better fit for the experimental data is obtained if a hard pomeron term is added to the soft pomeron in the amplitude of the process $\gamma p \rightarrow v p$.

This hard pole is attributed to the non hadronic phase of the photon. Thus according to Regge theory, the amplitude of this process is given as $[23,24]$

$$
A(s, t)=\sum_{i} X_{i} F(t) G(t)\left(\frac{s}{s_{i}}\right)^{\alpha_{i}(t)-1} e^{-\frac{1}{2} \pi \alpha_{i}(t)}
$$

The summation is over the exchanged reggeons, soft and hard pomerons. The parameters $X_{i}$ are the coupling of the exchanged trajectories to the interacting particles 
(residues). These parameters are fixed by the experimental data. The form factor $F(t)$ is the Dirac form factor for the proton, $G(t)$ is the $\gamma \rightarrow v$ transition form factor, $\alpha_{i}(t)$ are the Regge trajectories and $s_{i}$ are related to the slopes of the trajectories. All these factors are used as given in references [23-27] for both Rho and phi mesons amplitudes. For the virtual photon interaction $\gamma^{*} p \rightarrow v p$, the two pomeron model is also adopted but now the two components have $Q^{2}$-dependent residues.

The residues are fixed by fitting the experimental data. The formulas in $(1,4,5)$ are used to fit the experimental data [25] in Fig.(1) for the Rho and phi mesons at each value of $Q^{2}$. As a result the coefficients $X_{i}$ in the virtual photon case are obtained. In this fitting the coefficient of the reggeon term has been frozen at that of the real photon case in order to simplify the fitting procedure.

Here the coefficients of the hard and the soft pomerons at the different values of $Q^{2}$ are written in an another convention, that is the coefficients of the hard term and the soft term in the real photon case are multiplied by the factors $c_{0}$ and $c_{1}$ respectively. These factors are adjusted using the chi-square method until a good fit for the data is obtained. Our fitting also compared with that obtained using the $w^{\delta}$ method.

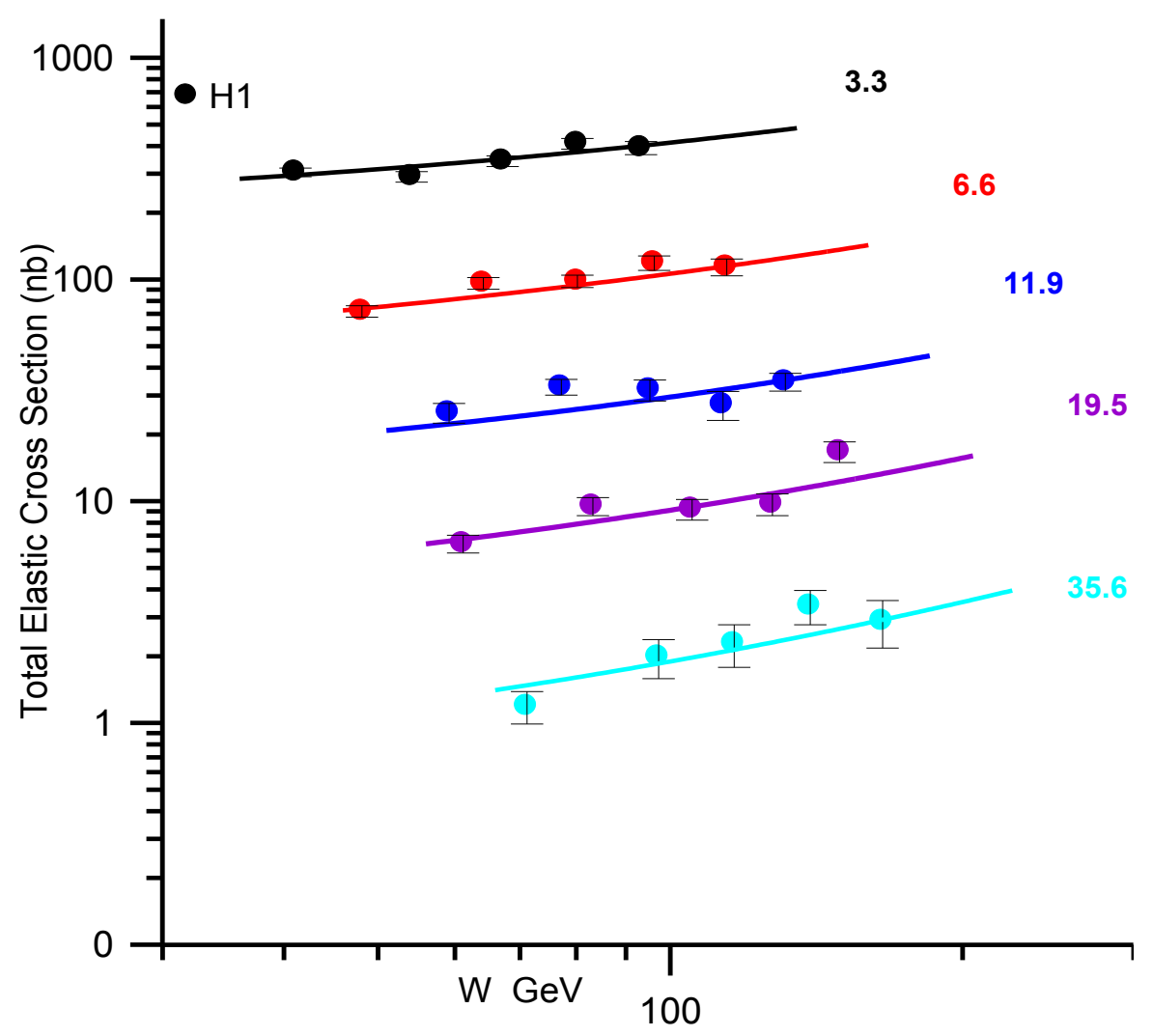

(a) 


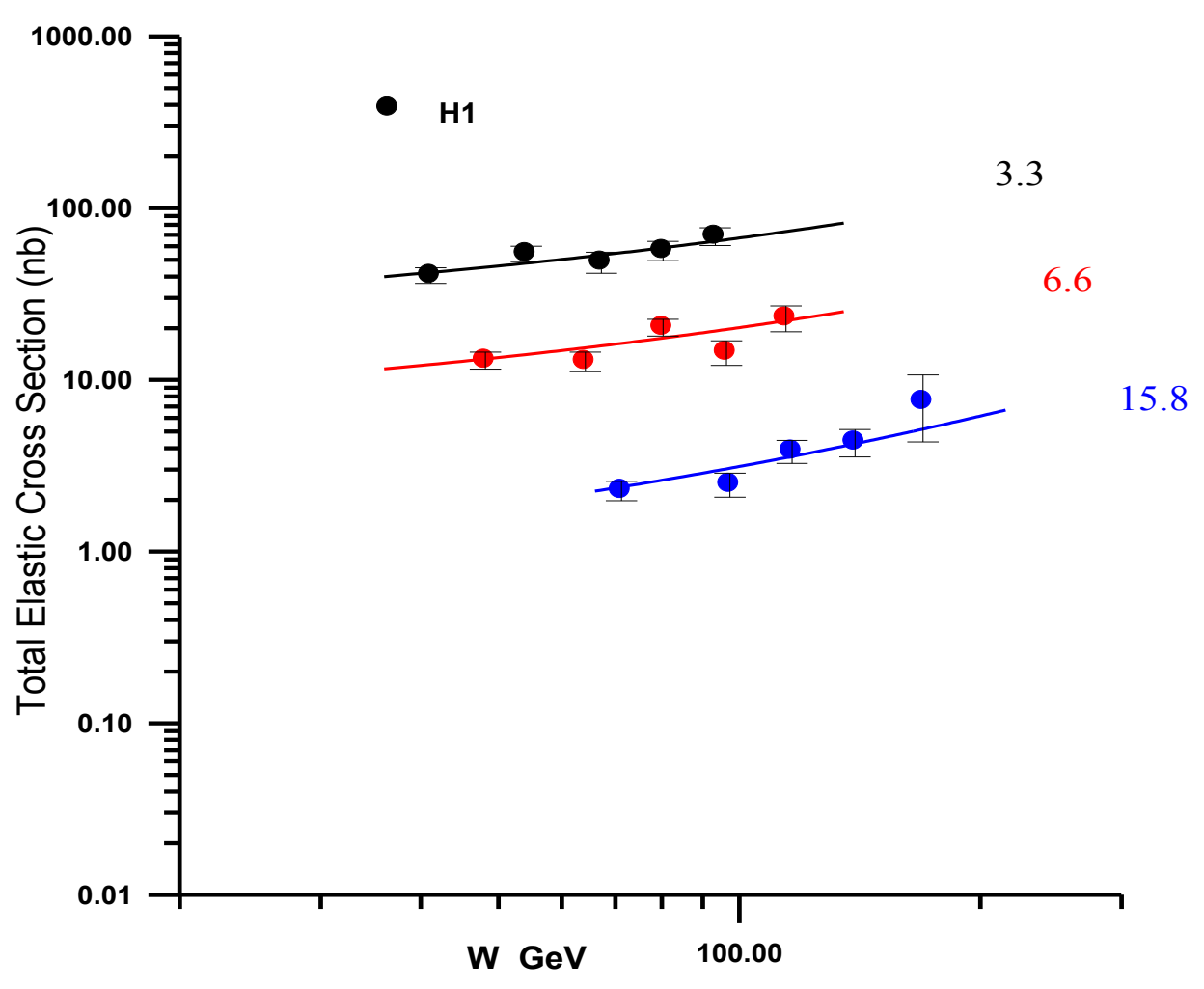

(b)

Fig. 1. Vector mesons electroproduction cross sections as a function of the energy (w) for different values of $\left(Q^{2}\right)$, (a) for Rho meson and (b) for Phi meson. The curves represent the predictions from the model in terms of eqs. (6-9).

These factors are denoted by the weights of the poles. The values of $c_{0}$ and $c_{1}$ of fitting the $\rho$ and $\varphi$ productions for different values $Q^{2}$ in Fig. (1) are tabulated in Tables (1) and (2) respectively. The hard pomeron weights $\left(c_{0}\right)$ for $\rho$ and $\varphi$ are plotted together in Fig. (2a) as a function of $Q^{2}$ while those of the soft pomeron weights $\left(c_{1}\right)$ are given in Fig. $(2 \mathrm{~b})$.

Table 1. Variations of the soft and the hard pomeron weights with $Q^{2}$ for Rho mesons.

\begin{tabular}{|c|c|c|c|c|c|c|}
\hline$Q^{2}$ & 0 & 3.3 & 6.6 & 11.9 & 19.5 & 35.6 \\
\hline$c_{1}$ & 1 & 0.94 & 0.88 & 0.86 & 0.8 & 0.68 \\
\hline$c_{0}$ & 1 & 2.75 & 3.1 & 4.2 & 4.5 & 5 \\
\hline
\end{tabular}

Table 2. Variations of the soft and the hard pomeron weights with $Q^{2}$ for Phi mesons.

\begin{tabular}{|c|c|c|c|c|}
\hline$Q^{2}$ & 0 & 3.3 & 6.6 & 15.8 \\
\hline$c_{1}$ & 1 & 0.64 & 0.62 & 0.5 \\
\hline$c_{0}$ & 1 & 2 & 2.2 & 4.9 \\
\hline
\end{tabular}




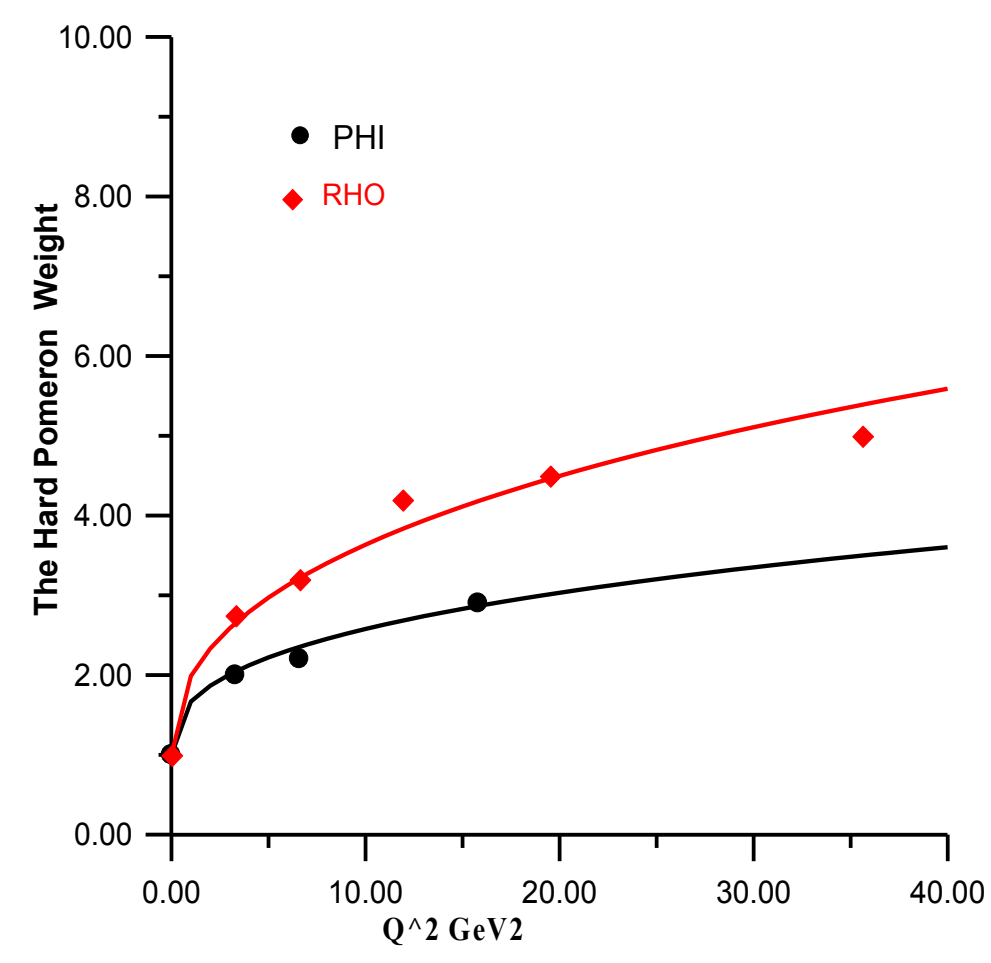

(a)

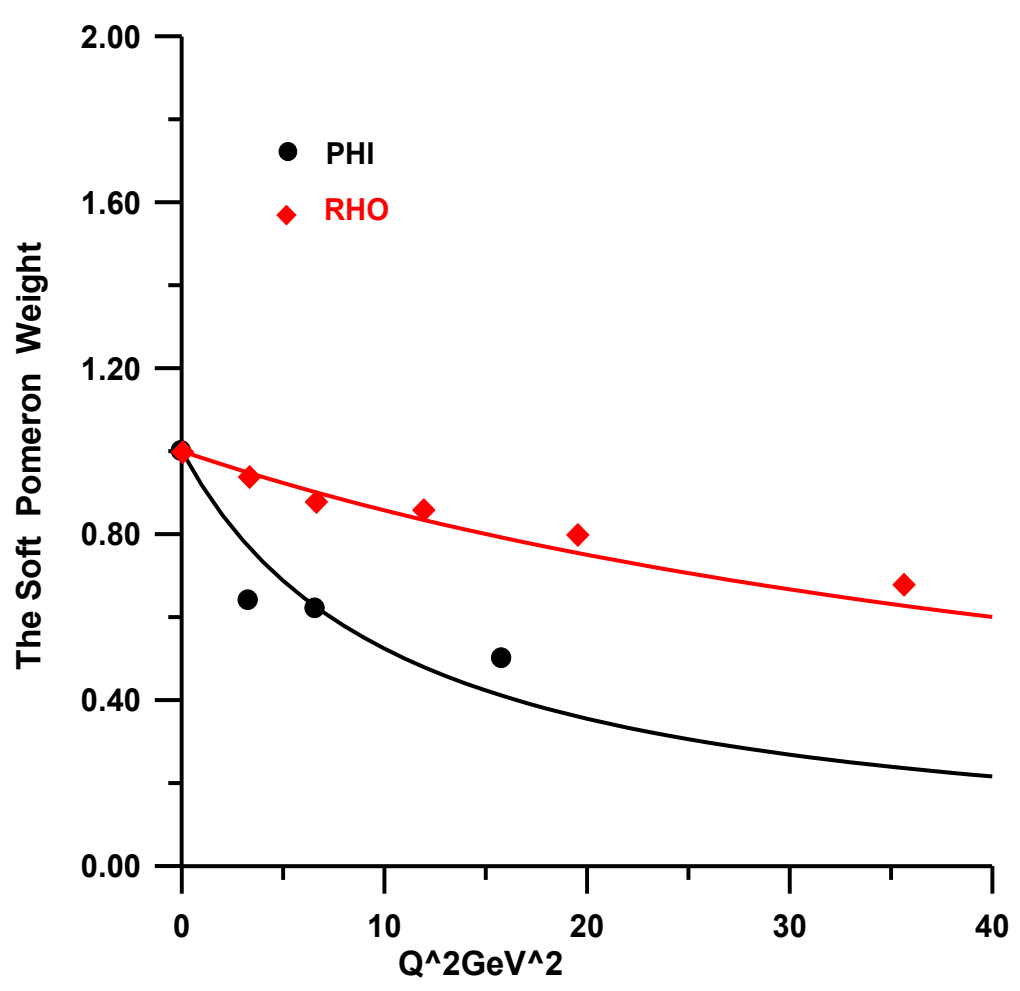

(b)

Fig. 2. a) hard pomeron weight and b) soft pomeron weight as functions of $Q^{2}$ for Rho and Phi meson productions. 
The following equations are used to fit the variations of the pomeron weights with $Q^{2}$ in Fig. (2):

For the Rho mesons

$$
\begin{gathered}
c_{1}=1 /\left(1+\frac{Q^{2}}{70}\right) \\
c_{0}=1+1.1\left(Q^{2}\right)^{0.45}-0.013 Q^{2}
\end{gathered}
$$

While for the Phi mesons

$$
\begin{gathered}
c_{1}=1 /\left(1+\frac{\left(Q^{2}\right)^{0.95}}{11}\right) \\
c_{0}=1+0.67\left(Q^{2}\right)^{0.38}-0.003 Q^{2}
\end{gathered}
$$

It is clear that the hard pomeron weights in Fig. (2a) for both mesons are increasing with $Q^{2}$, but the rate of the increase is decreasing with $Q^{2}$. Therefore, both curves should get to a region which on average is a constant with $Q^{2}$ at high values of $Q^{2}$. This arises from the behavior of the steepness of the cross sections in fig. (2). In the hardening region, the steepness of the cross section with energy should be constant as $Q^{2}$ increases. It is clear that the hard weights of the phi mesons reach the hardening region faster than that of the Rho mesons. As $Q^{2}$ increases the hard pomeron dominates the cross section while the contribution from the soft pomeron decreases. This means that the weights of the hard pomeron increase while that of the soft pomeron decrease as given by Fig. (2). The hard pomeron weights of the Rho mesons are above that of the phi meson because of the mass scale. This is consistent with expectations from the hard models as the hard scale involves both the $Q^{2}$ and the vector meson mass. As the Rho meson is in a softer regime then at the same $Q^{2}$ the cross section is higher than that of the Phi meson and $c_{0 \rho}>c_{0 \varphi}$. On contrary the $w^{\delta}$ distribution indicates that $\delta_{J / \psi}>\delta_{\varphi}>\delta_{\rho}$ since the $J / \psi$ production is a hard process at any value of $Q^{2}$. Using eqs. $(4,6,7,8,9)$ in (5), the fits in Fig. (1) for the data are obtained. It is clear that the extracted equations can reasonably reproduce the experimental data.

\section{DISCUSSION AND CONCLUSIONS}

Two pomeron model is used to analyze the electroproduction of low mass vector mesons. The soft and the hard pomeron were assumed to be Regge poles. Consequently, their Regge trajectories should be independent of the photon virtuality as Regge theory is dealing with on shell particles. Instead the coefficients of the poles or their weights are assumed to involve the virtuality. The weights are assumed to represent the coefficients at a given $Q^{2}$ divided by that at $Q^{2}=0$. Therefore, as the Rho meson is in a softer regime, the hard pomeron weight is higher than that of the Phi meson, while the $J / \psi$ hard pomeron is around one. To normalize the increase in the hard weights the soft weights are decreasing as in given in Fig. (2b). The weights are extracted by fitting the experimental data using chi-square method. This method works better for higher numbers of data points. Moreover, higher data points of the pomeron weights are needed especially at high values of $Q^{2}$. However, different 
formulae are obtained by fitting the weights points. Reasonable fits for the experimental data are achieved by involving these formulae in Regge amplitude. In this analysis, data from H1 collider are used. The extracted equations can be used to predict the cross sections at any other values of $Q^{2}$. However the ZEUS collider data may need slight adjustments in the parameters of the extracted equations.

\section{References}

[1] A. Donnachie, P. V. Landshof, Phys. Lett. B296 (1992) 227.

[2] S. J. Brodsky, L. Frankfurt, J. F.Gunion, A. H. Mueller, M. Strikman, Phys. Rev. D50 (1994) 3134.

[3] M. G. Ryskin, Z. Phys. C57 (1993) 80.

[4] H .G. Dosch, E. Ferreira, Eur. Phys. J. C51 (2007) 80.

[5] A. D. Martin, M. G. Ryskin, T. Teubner, Phys. Lett. B454 (1999) 339.

[6] J. Breitweg et al.(ZEUS Coll.), Eur. Phys. J. C6 (1999) 603.

[7] C. Adolf et al., Eur. Phys. J. C13, (2000) 371.

[8] L. P. A. Haakman, A. Kaidalov, J. H. Koch, Phys. Lett. B365 (1996) 411.

[9] L. L. Jenkovszky, E. S. Martynov, F. Paccanoni, Regge pole forvector meson photoproduction at HERA, hep-ph/9608384v1 (1996).

[10] Martynov, Predazzi, Prokudia, Phys. J. C26 (2001) 241.

[11] H1 Coll., Nucl. Phys. B 463 (1996) 3.

[12] H1 Coll. Eur. Phys. J. C 46 (2006) 5.

[13] H1 Coll., Phys. Lett. B 541 (2002) 251.

[14] H1 Coll., Phys. Lett. B 483 (2000) 23.

[15] ZEUS Coll., Eur. Phys. J. C 6 (1999) 603.

[16] ZEUS Coll., Phys. Lett. B 377 (1996) 259

[17] ZEUS Coll., Z. Phys. C73 (1996) 73.

[18] ZEUS Coll., Z. Phys. C75 (1996) 215.

[19] ZEUS Coll., "Exclusive Photoproduction of Upsilon mesons at HERA", presented at EPS2007, Manchester 2007.

[20] ZEUS Coll., PMC Phys. A (2007) 1.

[21] H1 Coll., Phys. Lett. B 539 (2002) 25.

[22] Khalid M. A. Hamad, Eur. Phys. J. C 72 (2012) 1927.

[23] A. Donnachie, P. V. Landshoff, Phs. Lett. B 478 (2000) 146.

[24] A. Donnachie, P. V. Landshoff, "Successful description of exclusive vector meson electroproduction" arXiv: hep-ph / 08030.686v1 (2008). 
[25] H1 Coll. "diffractive electorproduction of Rho and Phi mesons at HERA" arXiv: hep-ex/0910.5831v3 (2010).

[26] Yu. A. Baurov, International Letters of Chemistry, Physics and Astronomy 1 (2014) 41-51.

[27] Khalid M. A. Hamad, International Letters of Chemistry, Physics and Astronomy 5 (2014) 50-57. 